\title{
Redes de justicia ambiental en América Latina y las nuevas instituciones de lo común
}

Mauricio Berger*

\section{INTRODUCCIÓN ${ }^{1}$}

Uno de los intereses que ha orientado nuestra investigación ha sido y es la actualidad de las prácticas políticas desde los afectados directos e indirectos, desde las tramas solidarias y cooperativas entre diversas experiencias, conformando redes de luchas ambientales contra los agronegocios, la mega- minería, la construcción de mega-emprendimientos energéticos e infraestructura (represas, autopistas, expansión inmobiliaria).

A partir de identificar y entrar en contacto con experiencias como la red de Pueblos Fumigados en Argentina, la Asamblea Nacional de Afectados Ambientales de México (ANAA) y la Red Brasilera de Justicia Ambiental (RBJA), pudimos conocer e interactuar con algunos de sus protagonistas y espacios de trabajo y acción. Algunas de las preguntas que orientaban nuestra indagación por entonces fueron: ¿de qué forma estas redes contribuyen a poner en común significados, interpretaciones, marcos, estrategias, acciones en la defensa de derechos ambientales, es decir, en qué modo las redes potencian la acción de las luchas; ¿Cuáles son las condiciones de posibilidad y sostenimiento de las redes frente a la institucionalidad del sistema oficial- estatal? ¿Qué poder tienen las redes para potenciar las luchas y conflictos locales y regionales en América Latina?

\footnotetext{
* Doctor en Ciencias Sociales de la Universidad de Buenos Aires (UBA), Argentina. Actualmente, es investigador asistente del Consejo de Investigaciones Científicas y Técnicas (CONICET), con el proyecto sobre redes de gobernanza ambiental en América Latina. Es profesor asistente en el Instituto de Investigación y Formación en Administración Pública (IIFAP), de la Universidad Nacional de Córdoba, donde desarrolla el proyecto de investigación "Afectados ambientales: estructuras de sostén para la defensa pública y la movilización legal-institucional”.
} 
El presente trabajo reúne algunas reflexiones que surgieron de compartir estos interrogantes en los contextos de interacción en las redes. Se trata de reflexiones que intentan situar desarrollos teóricos en el pensamiento de la inteligencia colectiva de las redes de luchas ambientales.

En un primer punto, unas notas en torno al uso que realizamos del concepto de red para dar cuenta de una forma organizativa de las luchas., Seguidamente, la pregunta por cómo esa forma organizativa genera una institucionalidad política otra, propia de las luchas, y con características que nos remiten a la noción de instituciones de lo Común, principalmente en las coordenadas de la Teoría de la Multitud de Hardt y Negri. Finalmente, a modo de cierre, algunas consideraciones que provienen de una entrevista que realizamos en el marco de la investigación con Michael Hardt, y que nos proveen de algunas clave de lectura para seguir pensando las redes como instituciones desde una provocadora tarea: el entrenamiento político de nuestros afectos.

\section{CAPITALISMO DE REDES VERSUS REDES DE LUCHAS}

En lugar de remitirnos al tradicional análisis de redes sociales para cartografiar la configuración de las interacciones entre partes o nodos de la red, caracterizar atributos, intensidad y flujos de estos intercambios, el uso que hacemos del término red se vinculó en una primera instancia a la posibilidad de pensar una forma organizativa de las luchas ${ }^{2}$, de la que no diremos novedosa en relación a su propia historia, sino que la actualidad de las prácticas políticas en las coordenadas de la globalización capitalista es lo que le otorga relevancia y renovado interés ${ }^{3}$. La diferencia de los actores tradicionales del sistema político tales como partidos, sindicatos y porqué no decirlo, polémicamente, los propios movimientos sociales, delinean un límite para pensar la potencialidad, la creatividad, la inteligencia colectiva de estas luchas no sólo en contra del avance de la desposesión sino también contra la misma burocratización, modulación, bloqueo y parálisis de la acción política.

Ampliando sobre la conceptualización de las redes, en anteriores investigaciones lo abordamos desde la correspondencia de las formas de dominacion y la creatividad de las luchas. En este sentido, una referencia fue el analisis que realiza Castells sobre la tendencia historica propia del capitalismo contemporaneo, las funciones y los procesos dominantes en la era de la informacion estan generados por los flujos de capital y fuerza de trabajo, configurando un tiempo y espacio en los que las tecnologias de la informacion proporcionan la base material para la formacion de una estructura social, que el autor denomina "la sociedad red". Castells señala que una estructura social que se base en las redes es un sistema muy dinamico y abierto, susceptible de innovarse sin amenazar su equilibrio, por lo tanto las redes son instrumentos apropiados para una economia capitalista basada en la innovacion, la globalizacion; para el trabajo, 
los trabajadores y las empresas que se basan en la flexibilidad y la adaptabilidad. En este nuevo contexto, las relaciones de poder no se definen fundamentalmente dentro de los limites territoriales e institucionales de los estado- nacion o del sistema inter- estatal, sino que las sociedades estan siendo conformadas por multiples redes socioespaciales de poder superpuestas (financieras, militares, mafiosas, mediaticas, de opinion publica, de organizaciones de trabajadores, de solidaridad altermundista, etc.) y que interactuan simultáneamente a escala local, nacional o global, dando lugar al espacio de lo trans- nacional.

Dado que las prácticas y organizaciones sociales y politicas estarían estructuradas y estructurían a su vez esta dinámica, una clave para comprender las bases de la sociedad tanto como el cambio histórico se encuentra en la comprensión y reapropiación de estas multiples formas de ensamblaje, espacio de los flujos, territorios, multi y trans- escalaridad, en un marco de lucha.

En el capitalismo de redes ${ }^{4}$ la hegemonia opera cada vez mas en un marco de soberania dispersa, como también la caracterizan Negri y Hardt ${ }^{5}$, la red es un vocablo omnipresente en la globalización y denomina tanto una forma de organización social como una infraestructura de comunicación, con capacidad de combinar la organización establecida mediante reglas con la flexibilidad, la adaptabilidad, la descentralización y la dispersión espacial, por lo que las movilizaciones actuales han aprendido a no detenerse en los estados territoriales y sus fronteras, pero se encuentran con obstáculos y desacoples para actuar inter y trans- escalarmente en las nuevas geografías del capitalismo.

Los problemas debatidos son consustancialmente trans-territoriales y no pueden localizarse ni ser resueltos por un espacio nacional. Al mismo tiempo, la falta de poderes publicos globales que reconozcan y procesen democráticamente la formación de voluntad politica en contextos de globalizacion ${ }^{6}$ frente al poder de la governance global corporativa, entre otras presenta un escenario de incertidumbres y perplejidades que requiere, de parte de la inteligencia colectiva de las luchas un pensamiento acorde a esta complejidad.

Nos referimos por tal, a los contextos político- institucionales multiactorales, multi- escalares que atraviesan las experiencias que resisten el neoextractivismo ${ }^{7}$, una explotación a gran escala de los bienes comunes sobre la base de la desregulación y re- regulación proactiva hacia los intereses económicos de las corporaciones y gobiernos y en detrimento de las garantías y derechos colectivos, por un lado, y las renovadas estructuras del llamado eco- capitalismo. Ya sea en el marco de acuerdos y negociaciones por el Tratado de Libre Comercio de América del Norte en México ${ }^{8}$, del Plan de Aceleración del Crecimiento en Brasil ${ }^{9}$ o del Plan Estratégico Agroalimentario en Argentina ${ }^{10}$ el avance de la llamada acumulación por desposesión ${ }^{11}$ no distingue fronteras nacionales e institucionales sino que las subsume en las nuevas segmentaciones de una pretendida soberanía capitalista por sobre las soberanías de los estado- nación y de la soberanía de las luchas por derechos. 
En este contexto, y con los interrogantes previamente planteados, nuestro trabajo logró identificar como algunos de los principales rasgos distintivos de dichas luchas, su configuración rizomática en una amplia diversidad de organizaciones, colectivos, personas y personalidades que la componen: afectados, académicos, sindicatos, ONGs, movimientos de mujeres, comunidades indígenas y campesinas, profesionales, entre otros. Lejos del centralismo democrático de los movimientos, las redes definieron instancias de coordinación abiertas, colectivas y rotativas, como asambleas y encuentros regionales (Pueblos Fumigados), Colegiados Políticos y encuentros nacionales (RBJA), Consejo de Representantes y Asamblea Plenaria (ANAA), con esfuerzos cotidianos para hacer posible una horizontalidad práctica, que combine la posibilidad de deliberación y acuerdos colectivos básicos con la autonomía de cada nodo u organización integrante de la red.

La amplia diversidad compositiva se expresa en estas experiencias no sin conflicto, la multiplicidad de lenguajes y formas de vida que se encuentran no siempre pueden llegar a elaborar consensos sin la amenaza de ruptura o parálisis de la acción, pero al mismo tiempo la posibilidad de que esas diferencias se expresen renueva y coloca el desafío de una democratización permanente en las propias luchas.

Una comprensión de la diversidad de tácticas y estrategias ha permitido superar algunos obstáculos de la organización en lo que concierne a los problemas para definir conjuntamente planes de acción, mismo así la actuación en distintos contextos de interacciones o escalas de los problemas ${ }^{12}$ : local, nacional, regional, inter y transnacional.

En la búsqueda de herramientas conceptuales para adensar la comprensión de estas características de las redes, nos encontramos con desarrollos teóricos que inspiran a pensar en las mismas también como una emergente forma de institucionalidad política, de tipo móvil, nómada, inestable en la que componen sujetos diversos con capacidad de renovación y de invención de alternativas para tratar con problemas comunes. Ensayamos una puesta en diálogo de la experiencia de la institucionalidad otra de las redes con desarrollos teóricos sobre las instituciones de lo Común, en una hipótesis que afirma el poder coinstituyente de las luchas.

\section{2. ¿REDES COMO NUEVAS Y NÓMADAS INSTITUCIONES DE LO COMÚN?}

La trilogía "Imperio", "Multitud" y "Commonwealth"13 de Michael Hardt y Toni Negri puede ser leída como una sistematización de discusiones en la teoría y filosofía política y social (marxista, spinoziana, vitalista, biopolítica y post- estructuralista, y del pensamiento político en la vertiente del marxismo autonomista, el operaísmo italiano, el zapatismo y los movimientos contra la 
globalización y las distintas olas de protestas y acciones recientes en América Latina).

Este desarrollo teórico y práctico- político ha permitido conceptualizar la transición del esquema de la soberanía de los estado- nación hacia los procesos de desterritorialización y reterritorialización de actores, instituciones y procesos políticos en el mundo de la globalización capitalista. Los procesos de transnacionalización del capital conllevan una nueva forma soberana, la de una administración imperial (empresas y organismos transnacionales que ejercen el gobierno global) en la cual cada estado cumple una subordinada función de comando local.

La soberanía por lo tanto no se puede localizar en un estado o institución o un centro de poder sino que se trata de una soberanía dispersa, difusa, que opera distribuidamente en forma de redes: en la economía de servicios que es el nuevo motor del capital, el poder se organiza en los espacios de los flujos financieros, de información, migratorios, entre otros. Tampoco la soberanía estatal en este contexto sigue teniendo como reverbero la figura de la moderna soberanía popular.

Emerge en su lugar, para esta perspectiva, la Multitud, como una nueva subjetividad política, múltiple, diversa, que se expresa políticamente no por la toma del poder sino por su rechazo, por un estar en contra y una desobediencia radical como acción política. También por lo que se juega en las demandas de producción y reapropiación de lo común que ha sido expropiado y capturado por el capital, desde los bienes comunes "naturales" (tierra, agua, biodiversidad) al común social (saberes, arte y cultura, habilidades socio, comunicacionales y creativas) para los procesos de acumulación: el general intellect.

Este último concepto, tomado de Marx, refiere al saber social acumulado: información como saber hacer, conocimiento sistémico, o comprensión como potencial acción, conocimiento personal como trasfondo cultural de cada individuo y procesos de especialización individual como mecanismos de parcelación del conocimiento personal en función del conocimiento social ${ }^{14}$. La captura, modulación y explotación del general intellect es lo que configura las modalidad de un capitalismo cognitivo, es decir, un régimen de acumulación que se funda en la apropiabilidad y control del saber y del conocimiento social. Mientras que en el capitalismo industrial la aportación de cada individuo resultaba medible sobre la base de la cantidad de mercancía física co- producida en una unidad de tiempo dada, en el capitalismo cognitivo el proceso de acumulación tiene su origen en una estructura reticular, el conjunto de flujos y regulaciones que son capaces de generar una cooperación social sin la que éste no podría existir. La aportación al proceso de acumulación no se mide individualmente sino por la interdependencia que es capaz de desarrollar con otros nodos de una red: en el capitalismo cognitivo no hay productividad individual sino productividad social en red. De manera que el general intellect es tanto el eje del proceso de 
acumulación como el eje de las demandas de reapropiación de la Multitud para un proyecto revolucionario. En esta lucha global juega un papel clave la construcción de una institucionalidad propia de la Multitud, que también toma las formas y procesos de las redes y el trabajo cooperativo pero en clave de producción y reapropiación de lo Común: las instituciones de lo Común.

La recuperación de la noción de institución de su concepción más ligada a la órbita estatal, es un intento de pensar cómo sostener y expandir el poder constituyente de una multiplicidad de formas de vida y subjetividades en una espacio y temporalidad de relaciones autónomas, en éxodo con respecto a las relaciones de dominio que establecen tanto el Estado como el mercado. Hardt y Negri critican la acepción de la ciencia política y la teoría del derecho en la que la institución sirve a la fundación de un orden constitucional soberano, y revierten este punto al afirmar que las normas y obligaciones institucionales se establecen en las interacciones regulares y abiertas a un proceso de continua evolución, en el que las singularidades que componen la Multitud no transfieren sus derechos o poderes, y se oponen a la formación de un poder soberano.

Por su parte, Paolo Virno ${ }^{15}$, importante pensador de esta perspectiva, también argumenta a favor de la recuperación del concepto de institución como un concepto clave, en el marco de la crisis y metamorfosis de la forma Estado, para una política de la Multitud. Se entiende aquí las instituciones como los rituales que usamos para resolver las crisis de una comunidad, asumiendo que nuestras especies se protegen de la incertidumbre creando reglas para proteger su propia praxis. Entonces el debate no debería darse entre fuerzas institucionales y no institucionales, sino que deberíamos identificar aquellas instituciones más allá del monopolio de la decisión política encarnada en el Estado.

Consideramos que los elementos que provee esta perspectiva aportan a pensar el poder constiyuyente de las luchas y de las redes de luchas en América Latina, en tanto que sus acciones muestran los límites de las estructuras estatales para defender lo público y los derechos. Una institucionalidad propia sobre la base de la autoorganización, la autoformación, la capacidad de definir los propios temas y cursos de acción, e inclusive una propia estructura normativa, no implica el riesgo de la burocratización, ni la despolitización como señalan algunas posturas críticas a la noción de institución. Por el contrario, la recuperación del concepto de institución que nos aporta la Teoría de la Multitud revisada y ampliada a la vez con los aportes de las redes en América Latina nos provee un horizonte de reflexividad y creatividad para sostener y expandir la potencialidad política de experiencias que producen y defienden espacios autónomos.

Esta opción teórica y política no está exenta de problemas que derivan fundamentalmente del salto que existe entre la conformación de lo común y la autoadministración de lo común en sociedades complejas, de la toma de decisiones de forma democrática, la interacción de una multiplicidad de prácticas y en múltiples escalas, la institucionalización del reconocimiento y garantías de derechos, y la siempre conflictiva relación con el estado. 


\section{A MODO DE CIERRE}

Pensar en la institucionalidad otra de las redes de luchas por la Justicia Ambiental, basada en experiencias de auto- organización y auto- formación implica pensar las luchas como espacios capaces de generar sus propias estructurad de reglas, y por lo tanto no exentos del riesgo de burocratización y despolitización. Más aún, nuestra hipótesis de trabajo no desconce el salto enorme que hay entre la conformación de lo Común y la auto- administración de lo común en sociedades complejas. Precisamente por ello nuestras reflexiones concluyen en que no podemos pensar en una transformación a la escala de lo que pueden nuestros cuerpos, como punto de partida, como socialidad primaria que proyecte las instituciones de nuevo tipo.

Recuperamos fragmentos de una conversación con Michael Hardt ${ }^{16} \mathrm{~A}$ modo de proponer una clave de lectura para seguir pensando las redes como instituciones desde una provocadora tarea, acaso desde una (bio)política menor o una micropolítica, generalmente sub-teorizada: el entrenamiento político de nuestros afectos.

"Estoy pensando porqué necesitamos instituciones. En parte se trata de hacer los movimientos más fuertes ( $y$ más duraderos) pero la cuestión es- y es una dificultad también, y una apuesta- es que podamos construir instituciones que no sean rígidas y exclusivas. Pienso en ellas más como hábitos sociales que como instituciones, o sea, no las veo como un lugar sino como una forma de actuar a la volvemos $y$ mantenemos en el tiempo. Una parte de esto es en relación a la eficacia, necesitamos ser capaces de construir desde nuestros éxitos y resistir las fuerzas represivas contra nosotros.

(...) Al menos desde mi perspectiva no hay razón para asumir que espontáneamente vamos a juntar y tener relaciones amorosas $y$ pacíficas relaciones sociales. Lo que es requerido no es tanto normas y prohibiciones como un entrenamiento político de nuestros afectos, una forma en la que aprendamos juntos a auto-gobernarnos. Y eso me trae otra pregunta casi-institucional, sobre qué es lo requerido para la educación de nuestros afectos políticos. No estoy sugiriendo que vayamos a la escuela para eso, sino eso es en parte lo que está siendo realizado actualmente, cualquier activista reconoce que parte de tu experiencia en el activismo es el entrenamiento de los afectos políticos y una forma de aprender progresivamente cómo trabajar con otros, cómo resolver conflictos con otros, cómo resolver falta de entendimiento, cómo trabajar con otros inclusive desde el desacuerdo, todos estos son, en pequeña escala, tipos de entrenamiento social en auto-gobierno. Y eso es parte de lo que pienso que tiene que ser cualquier proyecto de auto-gobierno, la transformación de los afectos es una educación sobre cómo estar juntos". 
Compartimos el sentido de Hardt en torno a que la pregunta por cómo estamos juntos es una pregunta institucional, o que remite a preguntarnos por la educación de nuestros afectos en tanto que éstos potencian las composiciones; y por nuestros hábitos, en tanto que éstos las expanden en el tiempo.

\section{REFERENCIAS}

ACSELRAD, Henri. “Ambientalização das lutas sociais - o caso do movimento por justiça ambiental”, Revista Estudos Avancados, 24(68), 2010, pp.103-119.

ALMEIDA, Alfredo et al.. Capitalismo globalizado e recursos territoriais: fronteiras da acumulação no Brasil contemporâneo. Lamparina, Rio de Janeiro, 2009.

BERGER, Mauricio and CARRIZO, Cecilia. "Environmental Justice in Latin America”, Environmental Justice, 5(2), Special Issue on Environmental Justice in Latin America, Guest Editors: Mauricio Berger and Cecilia Carrizo Sineiro. New York: Mary Ann Liebert, Inc. Publishers, 2012. http://online.liebertpub.com/toc/env/5/2

BERGER, Mauricio. “Justicia ambiental en América Latina. Inteligencia colectiva y creatividad institucional contra la desposesión de derechos” En eCadernos Ces 17. Universidad de Coimbra Portugal, 2013. http://eces.revues.org/1128

CARRIZO, Cecilia y BERGER, Mauricio. "Multitudes ambientalistas en lucha contra los agrotóxicos”, en: Revista História em Reflexão, 7(13), Dossié Movimientos Sociales, 2013.

CASTELLS, Manuel: Comunicación y Poder, Madrid, Alianza Editorial, 2009. pp. 33 a 85.

FUMAGALLI, Andrea. Bioeconomía y capitalismo cognitivo. Hacia un nuevo paradigma de acumulación. Traficantes de Sueños, Madrid, 2010, pp 85 a 137.

GRAS, Carla y HERNANDEZ, Valeria. El Agro como Negocio. Producción, sociedady territorios en la globalización, Buenos Aires, Editorial Biblos, 2013, pp. 17 a 48 y 289 a 321.

GUDYNAS, Eduardo. “Diez tesis urgentes sobre el nuevo extractivismo”, en Autores Varios, Extractivismo, política y sociedad. Quito, Ecuador, CAAP (Centro Andino de Acción Popular) y CLAES (Centro Latino Americano de Ecología Social), 2010 pp. 187-225.

HARDT, Michael y NEGRI, Antonio. Imperio, Buenos Aires, Editorial Paidós, 2001, pp. 73 a 185.

HARDT, Michael y NEGRI, Antonio. Multitud. guerra y democracia en la era del Imperio, Buenos Aires, Editorial Debate, 2004, pp. 195 a 325.

HARDT, Michael y NEGRI, Antonio. Commonwealth, Cambridge, The Belknap Press of Harvard University Press, 2011, pp. 170 a 196.

HARVEY, David. El nuevo imperialismo, Madrid, Akal, 2004, pp. 111 a 160. 
MALERBA, Juliana. "Sustentabilidade como processo social: a experiência da Rede Brasileira de Justiça Ambiental” en Autores Varios (Red de Redes por la Justicia Ambiental en América Latina) Poder Constituyente y Luchas Ambientales. Hacia una red de redes en América Latina. Ediciones de Autor, SJA/UNC, 2014.

MEZZADRA, Sandro: "Extractivismo y política de lo común”. Sandro Mezzadra entrevistado por Clinämen y Maura Brighenti. En Sitio Web Lobo Suelto. Serie Nuevo Conflicto Social, 2013. http://anarquiacoronada.blogspot.com.ar/2013/12/serie-nuevoconflicto-social.html

ROSAS LANDA, Octavio. "La Asamblea Nacional de Afectados Ambientales como experiencia organizativa frente al desvío de poder del estado mexicano" en Autores Varios (Red de Redes por la Justicia Ambiental en América Latina) Poder Constituyente y Luchas Ambientales. Hacia una red de redes en América Latina. Ediciones de Autor, SJA/ UNC, 2014.

SCHLOSBERG, David. "Networks and Mobile Arrangements: Organisational Innovation in the US Environmental Justice Movement”. En Environmental Politics, 8 (1), 1995, 122-148.

Smith, Neil: “Geografia, diferencia y políticas de escala”. En Geografia Movimientos Sociais e Teoria, Sao Paulo, Terra Livre Publicação da Associação dos Geógrafos Brasileiros, 2002, pp. 127 a 146.

VIRNO, Paolo. Gramática de la Multitud. Para un análisis de las formas de vida contemporáneas, Buenos Aires, Editorial Colihue, 2003, pp. 75 a 187.

VIRNO, Paolo. “Antropología y Teoría de las Instituciones”, 2007. Traducción de Marcelo Expósito. http://eipcp.net/transversal/0407/virno/es.

Submetido: $29 / 06 / 2019$

Aceito: 28/10/2019 


\section{NOTAS}

1 Una versión de este texto fue publicada como capítulo, con el título "Redes de luchas como nuevas instituciones de lo común. Una conversación con Michael Hardt" en el libro Autores Varios (Red de Redes por la Justicia Ambiental en América Latina) Poder Constituyente y Luchas Ambientales. Hacia una red de redes en América Latina. Ediciones de Autor, SJA/UNC, 2014.

2 Schlosberg, 1995.

3 Castells, 2009.

4 Hartmann y Honneth, 2009.

5 Negri y Hardt, 2001, 2004.

6 Fraser, 2008.

7 Martinez Alier, 1995; Gudynas, 2009; Mezzadra, 2013.

8 Rosas Landa, 2014.

9 Malerba, 2014; Acselrad, 2010; Almeida, 2010.

10 Gras y Hernandez, 2009; Carrizo y Berger, 2013.

11 Harvey, 2004.

12 Smith, 2002.

13 Hardt y Negri, 2001; 2004; 2011.

14 Fumagalli, 2010.

15 Virno, 2003; 2007.

16 Entrevista completa puede leerse en Berger, Mauricio: "Redes de luchas como nuevas instituciones de lo común. Una conversación con Michael Hardt", capítulo del libro Autores Varios (Red de Redes por la Justicia Ambiental en América Latina) Poder Constituyente y Luchas Ambientales. Hacia una red de redes en América Latina. Ediciones de Autor, SJA/UNC, 2014. 


\title{
REDES DE JUSTICIA AMBIENTAL EN AMÉRICA LATINA Y LAS NUEVAS INSTITUCIONES DE LO COMÚN
}

\begin{abstract}
Resumen
El trabajo se inscribe en una línea de investigación sobre la actualidad de las prácticas políticas de los afectados ambientales, aquellas que conforman redes de luchas contra los impactos sanitarios y ambientales de los agronegocios, la mega- minería, la construcción de mega-emprendimientos energéticos y mega- infraestructura. A partir de identificar y entrar en contacto con experiencias como la red de Pueblos Fumigados en Argentina, la Asamblea Nacional de Afectados Ambientales de México (ANAA) y la Red Brasilera de Justicia Ambiental (RBJA), indagamos en torno a las formas en las que estas redes contribuyen a poner en común significados, interpretaciones, marcos, estrategias, acciones en la defensa de derechos ambientales,y qué poder tiene la forma organizativa de la red para potenciar las luchas y conflictos locales y regionales en América Latina. El artículo presenta algunas reflexiones situadas entre el aprendizaje de las redes y desarrollos teóricos, principalmente la Teoría de la Multitud de Hardt y Negri, y en estas coordenadas, una revisita al concepto de Instituciones de lo Común. La hipótesis tras estas indagacione empírico- conceptuales es la de pensar en la generación de una institucionalidad política otra, propia de la autonomía de las luchas, y en tal sentido post- soberana.
\end{abstract}

Palabras clave: Justicia ambiental. Neoextractivismo. Instituciones de lo Común. 


\title{
ENVIRONMENTAL JUSTICE NETWORKS AND THE INSTITUTIONS OF THE COMMONS
}

\begin{abstract}
The work is part of a line of research on the current political practices of those environmentally affected, those that make up networks of struggles against the health and environmental impacts of agribusiness, mega- mining, the construction of energy mega-enterprises and mega-infrastructure. By identifying and coming into contact with experiences such as the network of Fumigated Peoples in Argentina, the National Assembly of Environmental Affected People of Mexico (ANAA) and the Brazilian Network of Environmental Justice (RBJA), we investigate the ways in which these networks contribute to common meanings, interpretations, frameworks, strategies, actions in the defense of environmental rights. Also we inquiry what power the organizational form of the network has to strengthen local and regional struggles and conflicts in Latin America. The article presents some situated reflections between the learning of networks and theoretical developments, mainly from the Multitude Theory of Hardt and Negri, and in these coordinates, we revisit the concept of the Institutions of the Common. The hypothesis behind these empirical-conceptual inquiries is that of thinking about the generation of a different political institutionality, proper of the autonomy of struggles, and in such sense, a post- sovereign one.
\end{abstract}

Keywords: environmental justice- neo-extractivism- institutions of the Common. 